\title{
Reflexiones docentes acerca del diseño arquitectónico de los centros de formación profesional en Granada
}

\author{
Teaching reflections about the architectural design \\ of the vocational training centers in Granada
}

\author{
Luis Rodríguez ${ }^{1 \mathrm{a}}$, José L. Gallego ${ }^{2 \mathrm{~b}}$ \& Antonio V. Rodríguez ${ }^{2 \mathrm{~b}^{*}}$ \\ ${ }^{1}$ Consejería de Educación de Andalucía, Sevilla, España. \\ ${ }^{2}$ Universidad de Granada, Granada, España.
}

${ }^{a}$ Doctor en Arquitectura. Profesor de Formación Profesional. Consejería de Educación y Ciencia de Andalucía. ${ }^{b}$ Doctor en Pedagogía. Profesor titular del Dpto. de Didáctica y Organización Escolar. Facultad de Ciencias de la Educación. Universidad de Granada.

Recibido: $12-12-15$

Aprobado: 03-02-16

\section{*Correspondencia}

Email: arfuente@ugr.es

\section{Citar como:}

Rodríguez, L., Gallego, J., \& Rodríguez, A. (2016). Reflexiones docentes acerca del diseño arquitectónico de los centros de formación profesional en Granada. Propósitos y Representaciones, 4(1), 115-168. doi: http://dx.doi.org/10.20511/pyr2016. $\mathrm{v} 4 \mathrm{n} 1.88$

(C) Universidad San Ignacio de Loyola, Vicerrectorado de Investigación y Desarrollo, 2016. (c) BY-NC-ND Este artículo se distribuye bajo licencia CC BY-NC-ND 4.0 Internacional (http://creativecommons.org/licenses/by-nc-nd/4.0/). 


\section{Resumen}

Si se toma como medidor la especificidad del espacio arquitectónico en el ámbito educativo para valorar el protagonismo que a lo largo de la historia han tenido las enseñanzas, cabe destacar que la configuración del centro docente para la formación profesional, como escenario educativo de primer orden, que llega con medio siglo de retraso respecto a las escuelas, cuenta con escasos referentes que responden a un periodo muy concreto y que, en la actualidad, persisten excesivamente contaminados debido a la continua puja por la vinculación de estas con otros niveles educativos. Esta cuestión es la temática en la que se centra el presente artículo. Para ello, y recurriendo a la entrevista de docentes con experiencia, se valora: a) desde lo más conceptual, elementos y espacios arquitectónicos que deben caracterizar los centros de referencia; b) la pertinencia de los espacios dispuestos, tanto los que responden a requerimientos reglamentarios como a los criterios de los propios centros; c) hasta los aspectos más concretos sobre la manera de optimizar el espacio dispuesto para alcanzar una mayor amplitud curricular. Se concluye con una valoración muy positiva de la oportunidad de espacios precisos para estas enseñanzas, apostando con fuerza por aquellos que propician la libre relación del alumnado y permiten la permeabilidad entre las distintas disciplinas que convergen en el centro.

Palabras clave: Espacios arquitectónicos en la formación profesional, potencial del espacio en la generación de relaciones, permeabilidad en las aulas, Gran Taller.

\section{Summary}

If the specificity of the architectural space is considered as a measuring element in the education scope to value the prominence of teaching throughout history, it is worth mentioning that the configuration of the vocational training center as a first-rate education scenario that arrives half century of 
delay with respect to schools, has few references of very specific period. At present, they continue excessively contaminated because of the continued struggle for linking them with other education levels. This article is focused on this issue. To that end, by interviewing experienced teachers, we value: a) from the most conceptual aspect, elements and architectural spaces that should have those reference centers; b) the appropriateness of the arranged spaces, not only those that meet the regulatory requirements but those that meet the criteria of the centers; $c$ ) to the most specific aspects on the way to optimize the arranged space in order to achieve a larger curricular extent. It concludes with a high positive assessment of the opportunity of having the necessary spaces for teaching, relying more strongly on those favoring the free relationship of students and allowing the permeability among the different disciplines that converge in the center.

Keywords: Architectural spaces in vocational training, potential of space in the development of relationships, the permeability in classrooms, "Big Workshop". 


\section{Introducción}

Las singularidades de los programas pedagógicos emergentes, por las modificaciones que puedan suponer en la tarea docente, requieren atención continua sobre los espacios para adecuarlos a la función precisa a desarrollar en ellos, lo que para el arquitecto Rasmussen (2000) constituye el objetivo fundamental de la arquitectura. Desde el ámbito pedagógico, son muchos los autores que resaltan la importancia del Espacio Educativo. Es el caso de Viñao (2008), quien considera incuestionable el valor del espacio escolar por su dimensión educativa, por lo que justifica la estabilidad y la especificidad de los edificios educativos. Otálara (2010) va más allá, al entenderlo como el ambiente de aprendizaje o escenario de construcción de conocimientos y que, por tanto, deberá ser considerado como una herramienta o recurso más en la consecución del logro educativo. En la misma línea, la estructura arquitectónica que define el espacio escolar es, a juicio de Escolano (2000, como se citó en Rojas, 2011), determinante tanto en organización escolar como en el propio funcionamiento de la vida académica e, incluso, en los comportamientos que se generen en los ámbitos educativos. Desde esta misma perspectiva, son diversos los autores (Zabalza, 2002; Gairín, 2004; Lorenzo, 2005; Antúnez \& Gairín, 2009, entre otros) que vienen manifestando su interés por el espacio educativo, enmarcándolo dentro de la disciplina de la Organización Escolar como un recurso más a tener en cuenta. Por otra parte, desde el ámbito sociológico, Hernández (2010) alerta sobre la necesidad de considerar la habitabilidad de los espacios escolares para la generación de procesos eficaces de enseñanza-aprendizaje.

Aun así, algunos autores, como Toranzo (2007), convencidos de la necesaria afinidad entre arquitectura escolar y pedagogía, siguen acusando falta de protagonismo de los espacios escolares en el currículo. Esta preocupación por la concreción de los espacios educativos en las escuelas 
empieza a considerarse legalmente desde principios del siglo $\mathrm{XX}^{1}$, y llega a consolidarse durante el primer tercio del mismo, lo que conlleva, para estas enseñanzas, el fin de la reutilización de espacios originalmente diseñados para usos no educativos. Así, a partir de 1930 empiezan a recogerse los primeros frutos, que resultan de la conciliación entre arquitectura y pedagogía, con la aparición de un gran número de edificios escolares en España (Añon, 2015).

Este desarrollo arquitectónico escolar no se produce de la misma manera en todas las etapas educativas; en el caso de la Formación Profesional (FP), el recorrido histórico es relativamente corto (Gallego \& Rodríguez, 2011). La regulación e institucionalización de estas enseñanzas y, por tanto, el paso definitivo al centro educativo como lugar de formación, precisamente, no llegaría hasta finales del primer tercio del siglo XX, con el Estatuto de FP de 1928 (Rico, 2012). Sin embargo, esta ley, por su escasa afinidad con exigencias del mundo laboral, no tuvo mucho éxito (Cabrera, 1997). Por tal motivo, apenas dejó transcendencia en la configuración de los espacios de FP.

Con los Institutos Laborales, que surgen al amparo de la Ley de Bases de la Enseñanza Media y Profesional de 1949, arranca, con interés, la carrera en la confección de espacios apropiados para estas enseñanzas de FP. En estos edificios, que constituyen la primera referencia de nueva creación ${ }^{2}$ de la Arquitectura Escolar para la FP, destacan los talleres, además de las aulas tradicionales, consolidándose así una nueva especificación arquitectónica en estas construcciones escolares (Irles \& Pérez, 2012).

1 En 1905 se dictan las Instrucciones Higiénicas de construcciones escolares en España, y en 1920, el Ministerio de Instrucción Pública español crea una oficina técnica de construcciones escolares, desde la que se potencia la importancia del proyecto arquitectónico.

2 El hito más significativo lo encontramos en el Instituto Laboral de Daimiel, construido en el año 1951 por el arquitecto Miguel Fisac. Constituye un referente en las futuras escuelas de maestría industrial, e incluso del modernismo arquitectónico español, rompiéndose así con el monumentalismo arquitectónico tradicional. 


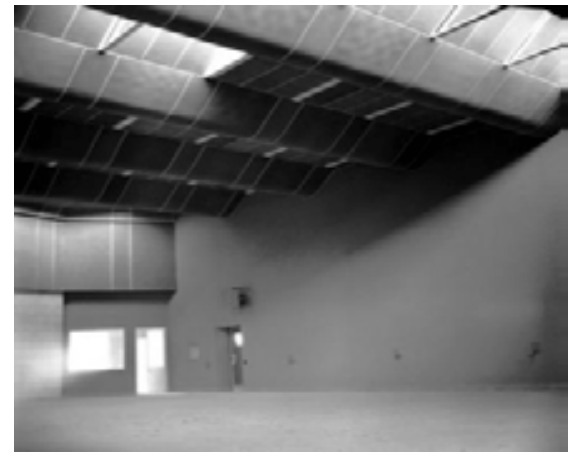

Figura 1. Taller del Instituto Laboral de Daimiel (extraída de Fundación Miguel Fisac http://fundacionfisac.org/ guia/?id=6).

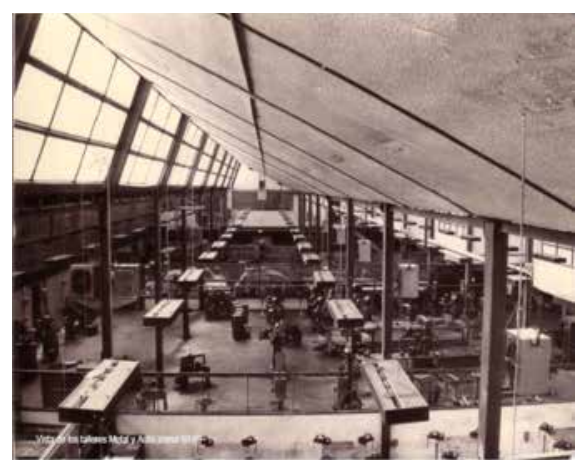

Figura 2. Taller de la Escuela de Maestría Industrial de Granada (cedida por el director del Ies H. Lanz).

El mayor punto de desarrollo arquitectónico se alcanza poco más tarde en las escuelas de Maestría Industrial que, reguladas por la Ley de FP Industrial de 1955 y como consecuencia, de un lado, del auge industrial del momento y, de otro, de la homogeneización de las enseñanzas de FP (Martínez, 2015), se expanden por todo el territorio nacional, en ocasiones como modelos arquitectónicos de gran trascendencia del movimiento moderno. Así, la especialización de infraestructuras propias de la FP adquiere una nueva dimensión que se acerca a la que ya existía en el resto de niveles educativos.

El marco legislativo que regula las intervenciones actuales sobre las enseñanzas de FP, en lo relativo al diseño de espacios arquitectónicos ante la necesidad de nuevos centros educativos o actuaciones sobre los existentes, distingue dos tipos de espacios. Por un lado, las instalaciones comunes ${ }^{3}$, entre las que se encuentran espacios como los despachos de dirección, de actividades de coordinación y de orientación, secretaría, biblioteca y sala de profesores, adecuadas al número de puestos escolares, además de los aseos y

3 Artículo 3 del Real Decreto 132/2010, del 12 de febrero, por el que se establecen los requisitos mínimos de los centros que impartan las enseñanzas del segundo ciclo de la educación infantil, la educación primaria y la educación secundaria, y artículo 9 del Real Decreto $1147 / 2011$, por el que se establece la ordenación general de la FP del Sistema Educativo. 
servicios ajustados a la normativa vigente. De otro, los espacios específicos ${ }^{4}$ o espacios propios ligados al currículo de cada título formativo, que vendrán definidos en los correspondientes Reales Decretos y tendrán como objetivo conseguir los resultados de aprendizaje de cada módulo profesional (Rodríguez, 2014).

El proceso metodológico seguido para la investigación que se pretende viene guiado por tres objetivos muy precisos, que tienen como finalidad última conocer en qué medida el diseño de los centros de FP puede favorecer el éxito educativo y, en este sentido, qué aspectos o criterios deberían tenerse en cuenta en los futuros proyectos arquitectónicos correspondientes. El punto de arranque lo conforman las siguientes cuestiones o interrogantes:

- ¿Qué consideración existe entre el profesorado de FP respecto al potencial del diseño de los edificios en los que desarrolla su labor educativa?

- ¿Los espacios dispuestos para estos centros se ajustan realmente a la especificidad de sus enseñanzas y alumnado?

De estas cuestiones surgen precisamente los objetivos operativos siguientes, que vertebran el objetivo general arriba determinado:

- Resaltar la importancia que otorgan los docentes al diseño de los centros en el proceso educativo, destacando los elementos arquitectónicos y los espacios de mayor pertinencia en el desarrollo de las enseñanzas de FP.

- Analizar y describir las opiniones que tienen los profesores sobre la adecuación de los espacios previstos por las disposiciones 
reglamentarias y los realmente dispuestos en los centros para las enseñanzas de FP.

- Valorar las necesidades sociales de interacción que pueden surgir entre el alumnado adscrito a las distintas enseñanzas que se ofertan en un mismo centro y recabar, entre los docentes, propuestas arquitectónicas que respondan a las mismas.

\section{Método}

Se trata de una investigación no experimental basada en un diseño transversal de corte eminentemente cualitativo, aunque los datos extraídos con la técnica de la entrevista semiestructurada en profundidad, que se describe más adelante, han sido sometidos a análisis cuantitativos, superando de esta manera la obsoleta guerra entre paradigmas (Díaz, 2014), apostando no solo por la combinación no simultanea de ellos, sino también por la integración simultánea de uno en el otro, con el único horizonte de lograr los objetivos propuestos (Gallego \& Rodríguez, 2016), para responder de esta manera complementada a la globalidad de la realidad estudiada, obteniendo un mayor rendimiento en la interpretación de los datos de la realidad que impulse una pertinente transformación de la práctica educativa (Salvador, 2001).

\section{Participantes: Población y muestra.}

La población la conforman todos los docentes que imparten enseñanzas de FP en ciclos formativos de grado medio y/o superior del contexto español, puesto que comparten la normativa arquitectónica legal para su construcción y dotación espacial. No obstante, la fuente para la extracción de la muestra se localiza en el Instituto de Enseñanza Secundaria Hermenegildo Lanz, centro de FP situado en Granada, por considerar que la variedad de la oferta educativa de esta etapa y el número de docentes que constituyen el claustro son lo suficientemente elevados para garantizar la representatividad del conjunto de 
la población (Rodríguez, 2014). Se selecciona así, estableciendo una serie de requisitos, un conjunto de profesores y profesoras que tienen conocimiento suficiente en el funcionamiento de los centros de formación profesional, de manera que las respuestas que proporcionen se ajusten a los objetivos planteados y que, por tanto, las conclusiones puedan ser extrapolables a otros centros similares. Así, como criterio de selección se plantean los siguientes requisitos: tener una antigüedad mínima de diez años como profesor de FP, haber prestado servicios como docente en al menos tres centros diferentes en los que se impartan enseñanzas de FP, y que la permanencia en el centro elegido como caso sea, como mínimo, de tres años consecutivos. Es, por tanto, un muestreo estratificado intencionado.

Además, otros de los requisitos impuestos, en este caso en el conjunto de la muestra, con el propósito de que las opiniones que se recaben sean lo más variadas $\mathrm{y}$, por tanto, generalizables posibles, fueron: de un lado, que estén representadas todas las familias profesionales del centro, y de otra, que la conformen los diferentes miembros que componen los distintos departamentos: jefes de departamento, profesores de enseñanzas secundaria y profesores técnicos de FP. Así, la muestra estuvo compuesta por 12 docentes: dos de cada una de las seis familias profesionales que se ofertan en el centro, donde uno de ellos es la persona que ostenta el cargo de jefe de departamento y están representados los dos cuerpos docentes, de manera que si aquel es profesor técnico de FP, el otro docente elegido pertenecerá al cuerpo de profesores de enseñanza secundaria, o a la inversa (Cfr. Tabla 1). 


\section{Tabla 1.}

Sujetos de la investigación.

\begin{tabular}{|c|c|c|c|c|c|}
\hline Código & Departamento & Cargo & $\begin{array}{l}\text { Experiencia } \\
\text { docente }\end{array}$ & $\begin{array}{c}\mathrm{N}^{\circ} \text { Centros } \\
\text { destino }\end{array}$ & $\begin{array}{c}\text { Años } \\
\text { Antigüedad } \\
\text { centro }\end{array}$ \\
\hline $\mathrm{J} 1$ & $\begin{array}{l}\text { Mantenimiento } \\
\text { de Vehículos } \\
\text { Autopropulsados }\end{array}$ & $\begin{array}{l}\text { Jefe de departamento/ } \\
\text { Profesor de Enseñanza } \\
\text { Secundaria }\end{array}$ & 22 años & 9 & 12 \\
\hline P1 & & Profesor Técnico de FP & 17 & 6 & 11 \\
\hline $\mathrm{J} 2$ & $\begin{array}{l}\text { Actividades } \\
\text { Físicas y } \\
\text { Deportivas }\end{array}$ & Jefe de departamento & 18 & 3 & 15 \\
\hline P2 & & $\begin{array}{l}\text { Profesor de Enseñanza } \\
\text { Secundaria }\end{array}$ & 28 & 3 & 20 \\
\hline $\mathrm{J} 3$ & $\begin{array}{c}\text { Edificación y Obra } \\
\text { Civil }\end{array}$ & $\begin{array}{l}\text { Jefe de departamento/ } \\
\text { Profesor de Enseñanza } \\
\text { Secundaria }\end{array}$ & 18 & 3 & 10 \\
\hline P3 & & Profesor Técnico de FP & 12 & 3 & 4 \\
\hline $\mathrm{J} 4$ & $\begin{array}{l}\text { Electricidad y } \\
\text { Electrónica }\end{array}$ & $\begin{array}{l}\text { Jefe de departamento/ } \\
\text { Profesor de Enseñanza } \\
\text { Secundaria }\end{array}$ & 15 & 4 & 14 \\
\hline P4 & & Profesor Técnico de FP & 22 & 5 & 7 \\
\hline $\mathrm{J} 5$ & Informática & $\begin{array}{l}\text { Jefe de departamento/ } \\
\text { Profesor de Enseñanza } \\
\text { Secundaria }\end{array}$ & 14 & 3 & 6 \\
\hline P5 & & Profesor Técnico de FP & 14 & 4 & 9 \\
\hline $\mathrm{J} 6$ & $\begin{array}{l}\text { Mantenimiento } \\
\text { y Servicios a la } \\
\text { Producción }\end{array}$ & $\begin{array}{l}\text { Jefe de departamento/ } \\
\text { Profesor de Enseñanza } \\
\text { Secundaria }\end{array}$ & 26 & 3 & 15 \\
\hline P6 & & Profesor Técnico de FP & 12 & 5 & 6 \\
\hline
\end{tabular}

\section{Instrumento de recogida de datos.}

Para recabar la opinión que tienen los docentes sobre el protagonismo y potencial de los espacios educativos en el proceso educativo, se ha procedido a realizar entrevistas guiadas a través de un cuestionario-guía previamente diseñado, lo que le confiere un carácter semiabierto. El documento se ha estructurado en dos partes (Anexo A):

- La primera, en la que se recogen los datos relativos a la experiencia docente del encuestado, para dilucidar que reúne los requisitos a 
los que se hace referencia en el apartado anterior, y el objetivo que se persigue con la entrevista, para facilitar el posicionamiento del sujeto.

- La segunda la componen distintas preguntas que guían el proceso, organizadas en tres bloques:

- El primero aborda las cuestiones más genéricas, relativas a los elementos arquitectónicos que, a juicio de los sujetos, deberían singularizar los espacios de los edificios escolares, con preguntas como:

¿Crees que las condiciones físicas del centro-edificio en el que tiene lugar la tarea educativa influyen en la consecución de los objetivos educativos establecidos?

¿QQué aspectos arquitectónicos consideras que son fundamentales en un centro educativo?

» ¿Qué espacios, además de los específicos de cada familia profesional, consideras que deberían distinguir un centro de FP respecto a cualquier otro centro de educación secundaria?

- A continuación, y con el objetivo de obtener de los participantes datos más precisos sobre los aspectos arquitectónicos que, en su opinión, tienen mayor relevancia en el proceso educativo, se fija la atención del sujeto en los espacios dispuestos en el centro de referencia y su influencia en los procesos educativos. Lo conforman las siguientes cuestiones:

¿Consideras que las características arquitectónicas de los espacios específicos dispuestos en los centros influyen en los resultados escolares? 
¿LLos espacios específicos que se fijan como mínimos para los ciclos formativos de tu especialidad son suficientes o, por el contrario, crees necesario algún otro espacio educativo?

¿LLos espacios educativos se diseñan con la suficiente flexibilidad para atender a los cambios legislativos o, por el contrario, condicionan el desarrollo del currículo?

- Por último, con el tercer bloque se pretende conocer el protagonismo que, para los encuestados, asume el espacio arquitectónico en el desarrollo de los aspectos transversales del currículo, a través de la potenciación de relaciones entre los principales actores del proceso de enseñanza-aprendizaje, y cuáles son, en su opinión, los requisitos que deben darse en los centros educativos para estas circunstancias. Las preguntas que se han realizado son las siguientes:

» ¿Qué opinas sobre la necesidad de que se establezcan relaciones interdisciplinares en los centros del FP y del protagonismo que, en este sentido, pudiera tener el diseño del edificio?

» ¿Qué criterios arquitectónicos crees que deberían tenerse en cuenta en los centros de FP para evitar el aislamiento que tiende a generarse entre los alumnos y alumnas de las diferentes enseñanzas?

Tanto las dimensiones como las cuestiones fueron validadas, en primera instancia, por el juicio de expertos (profesores del propio centro investigado y de la universidad donde se incluía la investigación), para lo que se utilizó una rúbrica de evaluación que fue debidamente realizada por cada uno de los expertos siguiendo diversos criterios, como longitud y duración, válido y generalizable, delicado y comprometido. En esta primera ronda de revisión se obtuvo un índice elevado de concordancia (por encima del 4, en 
una escala del 1 al 5), con un porcentaje de acuerdo igualmente elevado (por encima del 90\%). En segunda instancia, los guiones fueron validados en las primeras entrevistas experimentales, que también sirvieron para establecer códigos orientativos.

\section{Tratamiento y análisis de datos.}

Para analizar el contenido de las entrevistas, una vez transcritas, se ha generado previamente una serie de códigos o categorías (McMillan \& Schumaker, 2005; Stake, 2010) dada la falta de modelos teóricos de referencia sobre la dimensión escudriñada (Flick, 2004) que, teniendo como referencia los objetivos planteados, nos permitan el agrupamiento y la reducción de la información (Fernández, 2005; Tojar, 2006). Para ello se han seleccionado las entrevistas más relevantes, una por cada uno de los tres grupos representativos de los sujetos (jefes de departamento, profesores de enseñanza secundaria y profesores técnicos de FP). Tomadas como modelos, han sido analizadas en profundidad por expertos (profesores e investigadores) de diferentes contextos: enseñanza secundaria y universitaria.

Los códigos resultantes del proceso anterior se agrupan en tres niveles que se corresponden paralelamente con cada uno de los bloques en los que se organizan las preguntas del cuestionario (Cfr. Anexo I); esto es:

- Importancia que se les confiere a los elementos arquitectónicos en los espacios educativos de FP.

- Influencia que el espacio escolar dispuesto pudiera tener sobre los resultados de los procesos educativos, atendiendo al uso real que se hace de los mimos.

- Valoración de los espacios de uso común dispuestos en los centros o previstos en la normativa, atendiendo a su potencial como generadores de relaciones. 


\section{Resultados}

Siguiendo la estructura fijada en el apartado de metodología, se presentan los resultados que derivan de las entrevistas realizadas agrupados en tres apartados. El primero, que nos permite conocer cuáles son, en los centros de FP, de una parte, los aspectos arquitectónicos que gozan de mayor consideración entre el profesorado, y de otro, los espacios o usos de mayor pertinencia atendiendo a la singularidad de las enseñanzas y edades del alumnado en cuestión. A continuación se muestran los datos resultantes relativos a la valoración que hacen de los espacios que, de acuerdo con las disposiciones reglamentarias establecidas, tanto a nivel general, o espacios de uso común, como los que se vinculan a las distintas ofertas formativas, o espacios específicos, deben disponerse para el desarrollo de estas enseñanzas. En el tercer apartado se pone de manifiesto, a modo de síntesis, la importancia que le confieren al diseño arquitectónico de cara a mitigar o erradicar el aislamiento que tiende a generarse entre los diferentes miembros (profesorado y alumnado) adscritos a las enseñanzas o ciclos formativos ofertados en el centro educativo. Concluye con la exposición del análisis realizado sobre los criterios arquitectónicos que, tal como se manifiesta en las encuestas, son considerados fundamentales en los centros de FP para potenciar las relaciones entre sus usuarios.

\section{Importancia del espacio escolar en el proceso de enseñanza- aprendizaje.}

Respecto a la importancia de las características arquitectónicas del centro educativo, todos los profesores coinciden en destacar la trascendencia de las condiciones o características del espacio en el que se desarrolla la tarea educativa y, por tanto, la relación directa que existe entre estas y la consecución de los objetivos educativos fijados en los proyectos educativos. Para fundamentar sus respuestas, los sujetos hacen alusión a alguna o algunas cualidades arquitectónicas del espacio que consideran esenciales en estos centros (como, por ejemplo, la iluminación y la ventilación) y, en menor 
medida, a espacios concretos que deben formar parte de sus organigramas (como el salón de actos), cuestiones que se trataban en la segunda y tercera pregunta del cuestionario, respectivamente. Veamos, a continuación, los resultados obtenidos.

\section{Aspectos arquitectónicos de mayor relevancia para las enseñanzas de FP.}

El protagonismo que, según los docentes, tienen los diferentes aspectos arquitectónicos en el ámbito educativo de FP se reconoce tal como se recoge a continuación:

- La iluminación natural ha sido resaltada por la mayoría de los sujetos, nueve de los doce que componen la muestra (todos excepto J2, J3 y J6), esto es, el 75\% de los encuestados.

- La existencia de espacios y de dimensiones apropiadas se indica como pertinente en seis ocasiones, el 50\% del conjunto (sujetos P1, P5, P6, J1, J3, J4).

- En cuatro ocasiones se citan las condiciones térmicas (sujetos P3, P5, P6, J6), lo que representa el 33.3\% de la muestra.

- En tres ocasiones se señala la accesibilidad en el centro y la movilidad en el aula (sujetos P3, P4 y J5), coincidiendo en número con la ventilación natural (sujetos P4, P6 y J4), lo que representa el $25 \%$ de la muestra.

- La adecuada protección frente al ruido es apuntada dos veces (P3, J3), siendo el porcentaje correspondiente del 16.7\%.

- Tan solo en una ocasión se indica como aspecto relevante la orientación solar (sujeto J6), lo que representa tan solo el 8.3\% del profesorado.

- Existen dos aspectos, a los cuales se hacía alusión en el cuestionario, que son la versatilidad de los espacios y la distribución interior del aula, que no han sido considerados por ningún docente como relevantes en el proceso educativo. 
Para facilitar una visión comparativa de lo expresado anteriormente, se visualizan los resultados obtenidos (Figura 3), referidos a los aspectos que han tenido alguna representación:

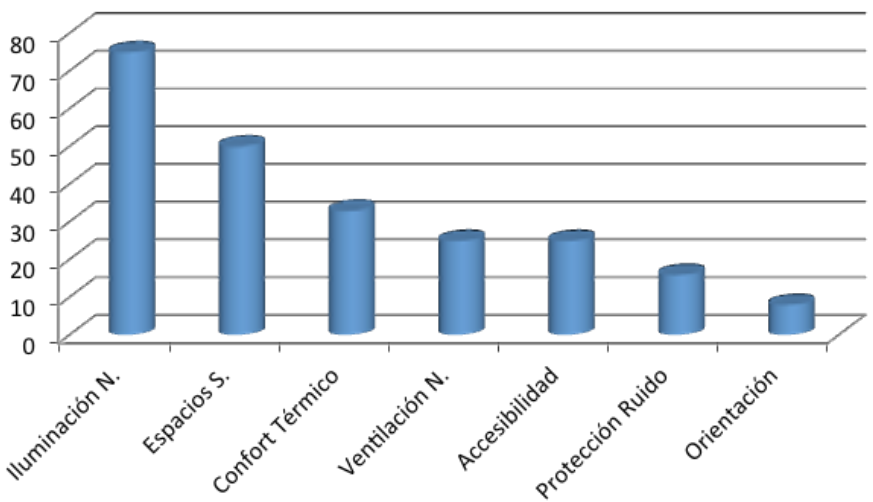

Figura 3. Aspectos arquitectónicos destacados por su mayor relevancia.

\section{Espacios arquitectónicos fundamentales en los centros de FP.}

Todos los encuestados se pronuncian afirmativamente sobre la necesidad de que los centros de FP dispongan de espacios arquitectónicos singulares que respondan a la especificidad de sus enseñanzas. Entre los espacios o usos concretos que deben figurar en los programas de estos centros deben incluirse, a juicio de los docentes, los siguientes: salón de actos, salas de informática de uso general, espacios para la libre relación de los alumnos, museo técnico y aulas especializadas. La importancia que se le concede a cada uno de estos espacios, atendiendo al número de veces que es indicado por los profesores, es, ordenada de mayor a menor, de la siguiente manera:

- El primer lugar lo ocupan las Aulas Especializadas, nombre que se les ha asignado a los espacios educativos adecuados o previstos para los currículos de las distintas enseñanzas. Estos han sido señalados por todos los sujetos, sin excepción, lo que representa el 100\% de la muestra.Un ejemplo de AE sería un laboratorio de pintura. 
- Nueve sujetos, el $75 \%$ de la muestra, coinciden en destacar la necesidad de disponer espacios para la libre relación del alumnado.

- La importancia de otros espacios, como el Museo Técnico, las Salas de Informática de uso general y el Salón de Actos, no ha sido muy resaltada pues tan solo se ha hecho referencia a cada uno de ellos en una ocasión, lo que representa sorprendentemente apenas el $8.3 \%$ del profesorado, dada la relevancia de su inclusión.

A modo de resumen, y con el objetivo de ofrecer una visión de conjunto, se recogen los datos anteriores en el gráfico siguiente (Figura 4).

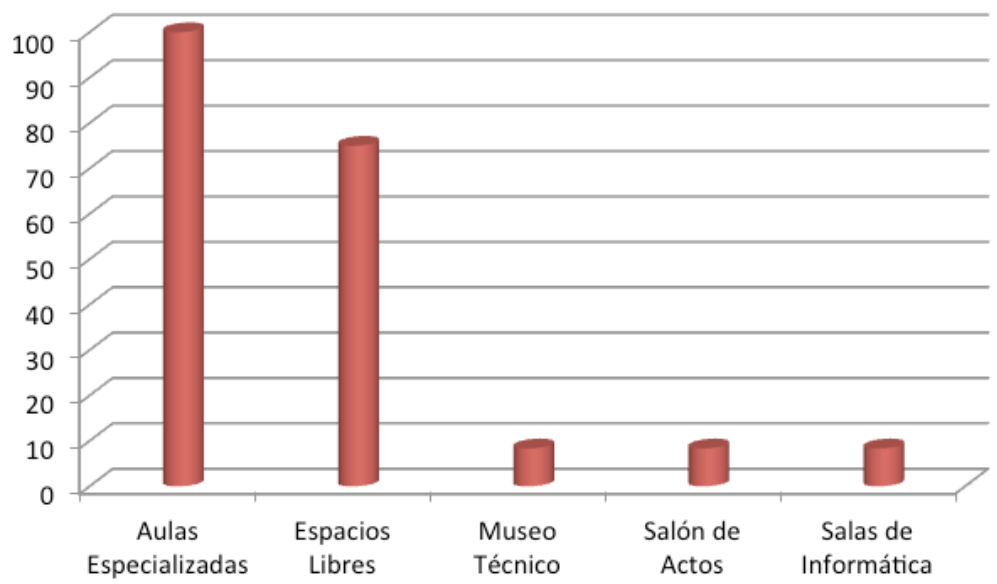

Figura 4. Espacios arquitectónicos destacados por pertinencia.

\section{Valoración de los espacios específicos dispuestos en los centros de FP.}

Las preguntas de partida que conforman el segundo bloque tienen como finalidad conocer la opinión de los sujetos sobre la relevancia de los espacios dispuestos en los centros educativos para el desarrollo de los currículos de las diferentes enseñanzas ofertadas en los mismos; esto es, los espacios específicos cuya pertinencia se puso de manifiesto en el apartado anterior. Se 
pretende así dilucidar sobre la manera en la que desde el diseño arquitectónico se puede favorecer o, en caso contrario, limitar la labor docente.

\section{Valoración de la influencia de las condiciones arquitectónicas de los espacios específicos dispuestos en los logros escolares.}

De los datos obtenidos puede deducirse que prácticamente la totalidad de los componentes de la muestra considera que las condiciones del espacio en que se desarrolla la tarea educativa son determinantes para el éxito escolar. Once de las doce respuestas han sido codificadas positivamente, lo que representa al $91.7 \%$ de los encuestados. No obstante, en las justificaciones que manifiestan surgen diferencias que pueden reunirse, por su similitud, en tres grupos:

- Ocho de los sujetos, el $66.7 \%$ del total, relacionan directamente las condiciones arquitectónicas con el confort del alumnado y, por ende, con el rendimiento escolar.

- Las dificultades que pueden surgir de cara al desarrollo del currículo, por la inadecuación de los espacios dispuestos con los contenidos específicos, es el principal inconveniente que resaltan dos de los docentes, el 16\% de la muestra.

- El sujeto restante se desmarca de los demás aludiendo, tras su afirmación, a cuestiones de naturaleza psicopedagógica que sobrepasan las que corresponden a las limitaciones curriculares expresadas por sus compañeros. No obstante, interesa destacar que este docente que se desmarca del conjunto, restando importancia a las condiciones arquitectónicas, para otorgársela, prácticamente de manera absoluta al docente, reconoce que hay elementos de partida que son esenciales en el proceso enseñanza-aprendizaje, apuntando expresamente hacia una adecuada iluminación en las aulas. 


\section{Valoración de las prescripciones establecidas por la normativa vigente sobre los requerimientos arquitectónicos en los centros de FP.}

Aunque con una ligera inclinación hacia el lado de la consideración como insuficientes, no existe un claro dominio, entre los encuestados, a la hora de valorar la pertinencia de los espacios arquitectónicos regulados por las disposiciones reglamentarias asociadas a las distintas ofertas educativas. De los once que, como se recoge en el apartado anterior, destacaron la importancia de los espacios dispuestos, el $60 \%$ de las respuestas ha sido codificado negativamente, el $20 \%$ positivamente, y el resto de las respuestas no ha sido codificado, principalmente por el desconocimiento o desinterés observado en los entrevistados sobre la regulación establecida al respecto.

Entre los que manifiestan su disconformidad con lo fijado por la norma, se generan dos grupos. La mayor representatividad, cuatro de los seis sujetos, viene de quienes consideran que la planificación prescrita es inadecuada por la falta de vinculación de los espacios previstos con el resto de elementos del currículo, principalmente contenidos y metodologías. Los sujetos restantes, dos, consideran que los currículos se modifican con cierta frecuencia y, sin embargo, los edificios no pueden asumir indefinidamente estas circunstancias, lo cual hace que se mantengan los requisitos espaciales de las disposiciones anteriores.

Tanto entre los sujetos que valoran positivamente lo recogido en la norma como entre los que restan importancia a lo que se prescribe en relación con la arquitectura escolar domina de manera rotunda, como problema principal, la desconsideración manifiesta desde los propios centros educativos y la propia administración hacia las cuestiones aquí tratadas. Como resultado, señalan, en la mayoría de las ocasiones se incumple de forma habitual lo dictado por las leyes españolas u otras disposiciones reglamentarias que las desarrollan. 


\section{Flexibilidad de los diseños arquitectónicos escolares para su adecuación a los proyectos educativos emergentes.}

A pesar de que en las respuestas precedentes quedó reflejada, en numerosas ocasiones, la opinión de los sujetos sobre la predisposición del espacio escolar dispuesto frente a las modificaciones curriculares, en este apartado se recoge expresamente el dominio que le corresponde entre la totalidad de la muestra para dar cumplida respuesta a uno de los objetivos planteados. Así, se les pidió a los sujetos que hiciesen una valoración general sobre la manera en la que se han encontrado los sitios docentes, que probablemente se diseñaron para un proyecto educativo muy diferente al que tienen que desarrollar. Se apunta como referencia, para llegar a un mayor nivel del caso elegido, el I.E.S. Politécnico Hermenegildo Lanz.

Existe una notable tendencia hacia la valoración negativa de la versatilidad de los espacios de FP a los nuevos planteamientos pedagógicos que incorporan los diferentes programas educativos. El 67\% de los sujetos encuentra los lugares preexistentes como un condicionante en el desarrollo de la práctica educativa, frente al $33 \%$ que manifiesta no haber encontrado dificultades en el desarrollo del currículo en ningún momento por las condiciones propias del contenedor de las actividades.

Entre las ocho respuestas que se han codificado negativamente se destaca, de forma contundente, la escasa analogía entre el espacio existente y las nuevas tendencias pedagógicas versátiles de los mismos. De forma expresa, en tres ocasiones, se alerta de la necesidad de readaptar la infraestructura arquitectónica ante la implantación de nuevos programas educativos. De lo contrario, afirman, esta asume el protagonismo curricular condicionando los planteamientos pedagógicos, lo que no favorece el éxito en lo implantado.

De otro lado, los otros cuatro sujetos restan importancia al aspecto arquitectónico, a favor de una inmediata dotación de recursos más adecuada 
a las nuevas exigencias educativas. Esta solución se presenta, dentro de este grupo, como la única posible para romper con la metodología dominante, que manifiestan como el principal condicionante pedagógico.

\section{El protagonismo del diseño arquitectónico en las relaciones del alumnado.}

Todos los docentes son conscientes de que tiende a generarse un elevado grado de aislamiento entre el alumnado de las diferentes familias profesionales como consecuencia de la falta de afinidad que existe entre sus elementos curriculares, a pesar de que las diferentes ofertas confluyan en el escenario o espacio arquitectónico.

\section{Valoración de la importancia que tiene la generación de relaciones sociales en los centros de FP en la formación del alumnado.}

Existe un consenso prácticamente absoluto al valorar la importancia que para la formación del alumnado de FP tiene el que se establezcan relaciones interdisciplinares entre los diferentes estudiantes de estos centros. Todas las respuestas de la muestra, salvo una, han sido codificadas positivamente, lo que supone un dominio del 91.7\%.

Los docentes que valoran positivamente la necesidad de fomentar las relaciones interpersonales en los centros de FP coinciden en su totalidad a la hora de justificar su postura, que responde a cuestiones vinculadas al currículo oculto. Además, en cuatro ocasiones, el $36.7 \%$ de este subconjunto se apunta con interés a la buena repercusión que pudiera tener el contacto fluido entre el alumnado de cara a las futuras decisiones formativas, al poder conocer directamente, y en profundidad, a través de las opiniones de sus compañeros, otras ofertas educativas. 
El docente que discrepa de sus compañeros justifica su posición anteponiendo la necesidad de desarrollar el currículo establecido que, a su juicio, está excesivamente ajustado al horario escolar y no permite, por lo tanto, que el alumnado disponga de tiempo para relacionarse libremente.

\section{Criterios arquitectónicos aportados como soluciones para evitar el aislamiento del alumnado en los centros de FP.}

Siguiendo la línea trazada en el apartado anterior, se les pidió a los sujetos su opinión acerca de la importancia que el diseño arquitectónico pudiera tener en la generación de relaciones en los centros de FP. Todos coincidieron en destacar el potencial del espacio arquitectónico dispuesto, señalándolo como protagonista principal.

Por último, para obtener unas conclusiones que puedan llevarse a la práctica a modo de criterios arquitectónicos a tener en cuenta en los diseños de los centros de FP, respondiendo así a uno de los objetivos fijados, se les preguntó sobre los espacios que deberían disponer o la manera en la que deberían relacionarse los existentes para favorecer la generación de relaciones. Todos hicieron alguna aportación, salvo uno, quien alegó no saber cómo podría solucionarse la cuestión planteada. Del análisis de las respuestas, existen algunas propuestas que se repiten en mayor o menor medida. Véanse a continuación en su orden jerárquico:

- La mayor permeabilidad de los espacios asociados a las distintas familias profesionales, especialmente aquellos en los que tienen cabida las enseñanzas más prácticas. Esta solución apunta a la idea del Gran Taller, y cuenta con un respaldo de cuatro sujetos, el 33.3\% del conjunto.

- El acercamiento entre familias profesionales a través de la centralización de alguno de los espacios específicos asignados a cada una de ellas, de manera que existan nexos de unión o espacios comunes para el alumnado de las distintas enseñanzas, es indicado como la mejor solución por dos docentes, representando tan solo el $16.7 \%$ de la muestra. 
- Cinco de los doce docentes, el $41.7 \%$, suponen que el aislamiento se evitaría disponiendo de un gran espacio central o común para todo el centro, que cuente con el potencial suficiente para la atracción de los flujos cotidianos del alumnado en el centro.

A continuación se presenta el conjunto de los datos que resultan del análisis efectuado con anterioridad (Figura 5):

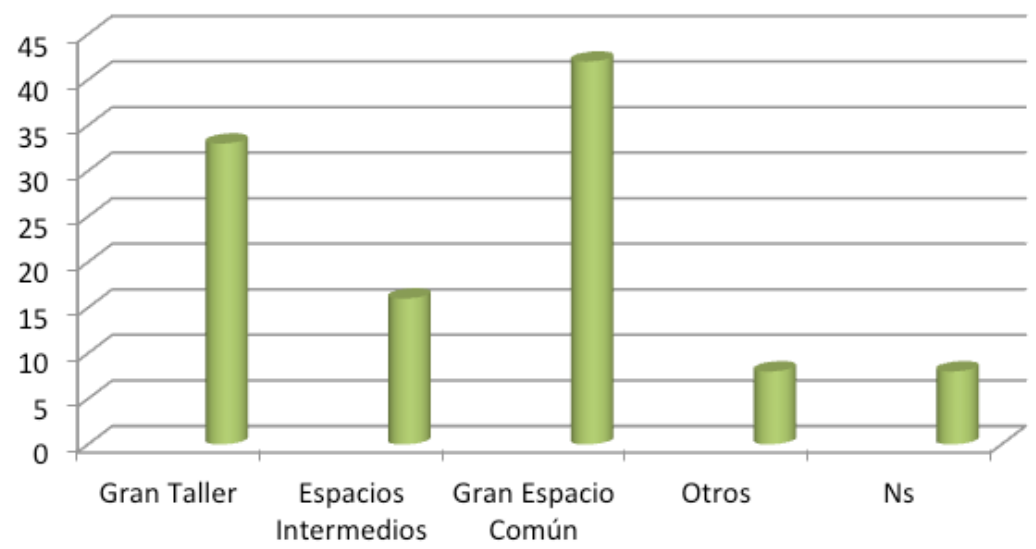

Figura 5. Criterios arquitectónicos planteados por los sujetos como soluciones para evitar el aislamiento en los centros de FP.

\section{Conclusiones}

Este estudio nos ha permitido conocer las creencias que tienen los docentes sobre la importancia de los espacios en el proceso educativo. Para ello se ha valorado cuáles han de ser los aspectos y espacios arquitectónicos de mayor relevancia en este centro; la consideración que sobre estas cuestiones se tiene tanto desde la administración, a la hora de dictar las resoluciones que regulan los espacios para el desarrollo de las enseñanzas ofertadas, como desde los propios centros educativos, en las disposiciones correspondientes; y por último, el protagonismo del diseño arquitectónico de cara a evitar el aislamiento entre el alumnado que, dentro del mismo centro, no comparte 
contenidos curriculares, culminando con la recogida de propuestas precisas para ello.

Con referencia al primer apartado, los docentes mantienen la misma concepción en líneas generales: las condiciones del escenario en el que se desarrollan las enseñanzas de FP son determinantes en el proceso educativo. En el diseño de este tipo de centros, el aspecto arquitectónico que más preocupa es la iluminación natural, seguido, en orden jerárquico, de los espacios adecuados a las enseñanzas, el confort térmico, la ventilación y la accesibilidad. Y en cuanto a los espacios requeridos, el protagonismo lo adquieren las aulas especializadas, por cuanto resultan adecuadas a los contenidos curriculares, y los lugares para la libre relación del alumnado, por cuanto actúan como intercambiadores de incalculable valor.

En lo que respecta a los espacios específicos dispuestos en el centro para cada una de las enseñanzas, puede aseverarse, tras las declaraciones docentes, que de ellos depende, en gran parte, el éxito escolar, y esto, principalmente, por su relación con el confort del alumnado. Existe una ligera inclinación hacia la valoración de los espacios específicos, que desde las disposiciones reglamentarias se asocian a las diferentes ofertas educativas, como poco pertinentes. La falta de afinidad entre los espacios regulados y las metodologías emergentes, y la influencia que ejerce el espacio preexistente, ante la escasa flexibilidad de los edificios prediseñados, son -en ese ordenlas causas de la consideración anterior. Sí se evidencia un alto grado de acuerdo en relación con la poca preocupación que se presta, desde los centros educativos, al cumplimiento de los requisitos fijados en las normas y disposiciones legales vigentes, $y$, en general, al escaso control que existe al respecto desde la administración educativa española.

Por último, sobre las relaciones personales y/o sociales en los centros de FP, cabe inferir que tiende a generarse un gran aislamiento asociado a las distintas ofertas formativas, como consecuencia de las 
diferencias curriculares, y que el diseño arquitectónico pudiera ser pieza clave para evitar esta tendencia. Para ello se apuntan distintas soluciones, a modo de criterios arquitectónicos, y a pesar de que no se da un predominio contundente destacan, estructuradas en función del número de repeticiones, un gran espacio común en el centro, la mayor permeabilidad de los espacios específicos “Gran Taller” y múltiples espacios de relación entre los diferentes espacios específicos.

\section{Discusión}

No se han encontrado datos que se centren de una manera exclusiva en la valoración de los espacios en los centros de FP. Sin embargo, no podemos decir lo mismo en otro tipo de instituciones, alcanzándose un mayor nivel de desarrollo en las escuelas de educación primaria. No obstante, atendiendo a las cuestiones más genéricas, pueden extraerse datos de otros autores susceptibles de ser comparados con los que resultan de esta investigación, predominando, en todos los casos, una clara tendencia hacia la convergencia. Es el caso de Toranzo (2007), que tras poner en cuestión los criterios de diseño escolares dominantes, caracterizados por concebir el aula como espacio principal y relegar a un segundo plano los espacios libres, concluye destacando la necesidad de generar espacios más dinámicos, frente a aquellos que tienden al encerramiento, por el potencial educativo que aportan. Rojas (2011), pese a que sus estudios se centran en otros niveles educativos, alerta de los riesgos que supone limitar los espacios escolares solamente a aulas, ante el escaso potencial de estas para la generación de interacciones significativas. También Arias (2013) destaca la necesidad de disponer espacios de participación y expresión como forma de combatir los ambientes de represión que se generan en los centros educativos por la falta de interacción física. 


\section{Referencias}

Antúnez, S., \& Gairín, J. (2009). La organización escolar. Práctica y fundamentos. Barcelona: Graò.

Añon, R. M. (2015). Inicios, evolución y decadencia del espacio arquitectónico en la Escuela Primaria Pública española. Cabás 13, 113-134.

Arias, M. (2013). La arquitectura escolar como espacio sociofisico formativo: una mirada desde los/as estudiantes (Tesis de maestría). Recuperado de http://tesis.uchile.cl/bitstream/handle/2250/115408/ Tesis.pdf? sequence $=1$

Cabrera, L. J. (1997). La FP en España antes de la Ley General de Educación de 1970. Revista de Educación, 312, 173-190.

Díaz, S. M. (2014). Los Métodos Mixtos de Investigación: Presupuestos generales y aporte a la evaluación educativa. Revista Portuguesa de Pedagogía, 48(1), 7-23.

Fernández, A. (2005). Métodos para evaluar la investigación en psicopedagogía. Madrid: Síntesis.

Flick, U. (2004). Introducción a la investigación cualitativa. Madrid: Morata.

Gairín, J. (2004). La Organización Escolar: contexto y texto de actuación. Madrid: La Muralla.

Gallego, J. L., \& Rodríguez, A. (2011). La Formación Profesional en España: historia y actualidad. Revista Educaçao Skepis, 2(3), 2045-2105. Recuperado de http://www.academiaskepis.org/revistaeducacao.html

Gallego, J. L., \& Rodríguez, A. (2016). La alteridad en educación. Madrid: Pearson.

Hernández, J. M (2010). Habitabilidad educativa de las escuelas. Marco de referencia para el diseño de indicadores. Revista Sinéctica, 35, 1-14.

Irles, R., \& Pérez, R. (2012). La documentación gráfica de los proyectos de los Institutos Laborales de Rafael Aburto Renovables en Elche y en Orihuela. En Actas del XI Congreso Internacional de Expresión Gráfica aplicada a la Edificación. Recuperado de http://www. academia.edu/3543144/Propuesta_docente_para_Analisis_de_Formas Arquitectonicas_Conceptualizacion_a_partir_de_los_movimientos_ artisticos_del_s._XX.

Lorenzo, M. (2005). Organización escolar. La construcción de la escuela como ecosistema. Madrid: MEC-Morata. 
Martínez, M. J. (2015). Consideraciones a una ley paradigmática en la historia de la formación profesional española: la Ley de Formación Profesional Industrial (FPI) de 1955. Participación Educativa, 4(6), 107-111.

McMillan, J. H., \& Schumacker, S. (2005). Investigación educativa. Una introducción conceptual. Madrid: Pearson.

Otálara, Y. (2010). Diseño de Espacios Educativos Significativos para el desarrollo de competencias en la infancia. Colombia: Universidad del Valle.

Rasmussen, S. E. (2000). La experiencia de la arquitectura. Barcelona: Reverté, S.A.

Rico, M. L. (2012). La enseñanza profesional y las clases medias en España (1924-1931). Revista Española de Historia, 240, 119-146.

Rodríguez, L. (2014). Estudio de los espacios arquitectónico-educativo en la Formación Profesional. El caso del I.E.S. Hermenegildo Lanz (Tesis doctoral). Universidad de Granada, España.

Rojas, C. (2011). El espacio aula como constructor de emociones y sentidos (Tesis de licenciatura). Universidad Académica Cristiana Santiago de Chile, Santiago de Chile. Recuperado de http://bibliotecadigital. academia.cl/bitstream/handle/123456789/914/tpdif17.pdf;jsessionid=E AD28834C04A76D28AF4AF2B9609F213? sequence $=1$

Salvador, F. (2001). Educación Especial. Enfoques conceptuales y de investigación. Granada: GEU.

Stake, R. (2010). Investigación con estudio de caso. Madrid: Morata.

Tojar, J. (2006). Investigación cualitativa: comprender y actuar. Madrid: La Muralla.

Toranzo, V. (2007). ¿Pedagogía vs. Arquitectura? Los espacios diseñados para el movimiento. (Tesis de maestría). Universidad de Buenos Aires, Buenos Aires. Recuperado de https://docs.google.com/document/ d/1 agpvb5PLS2uX_RjtVhdfYWJ13wBChXz9N4uGHqVKJIw/ edit?pli=1

Viñao, A. (2008). Escolarización, edificios y espacios escolares. Revista Participación Educativa, 7, 16-27.

Zabalza, M. A. (2002). Las relaciones interpersonales en las organizaciones. En Actas del VII CIOIE. País Vasco: Servicio de Publicaciones del País Vasco. 


\section{Anexo A. Guión de entrevista abierta para los participantes en la} investigación.

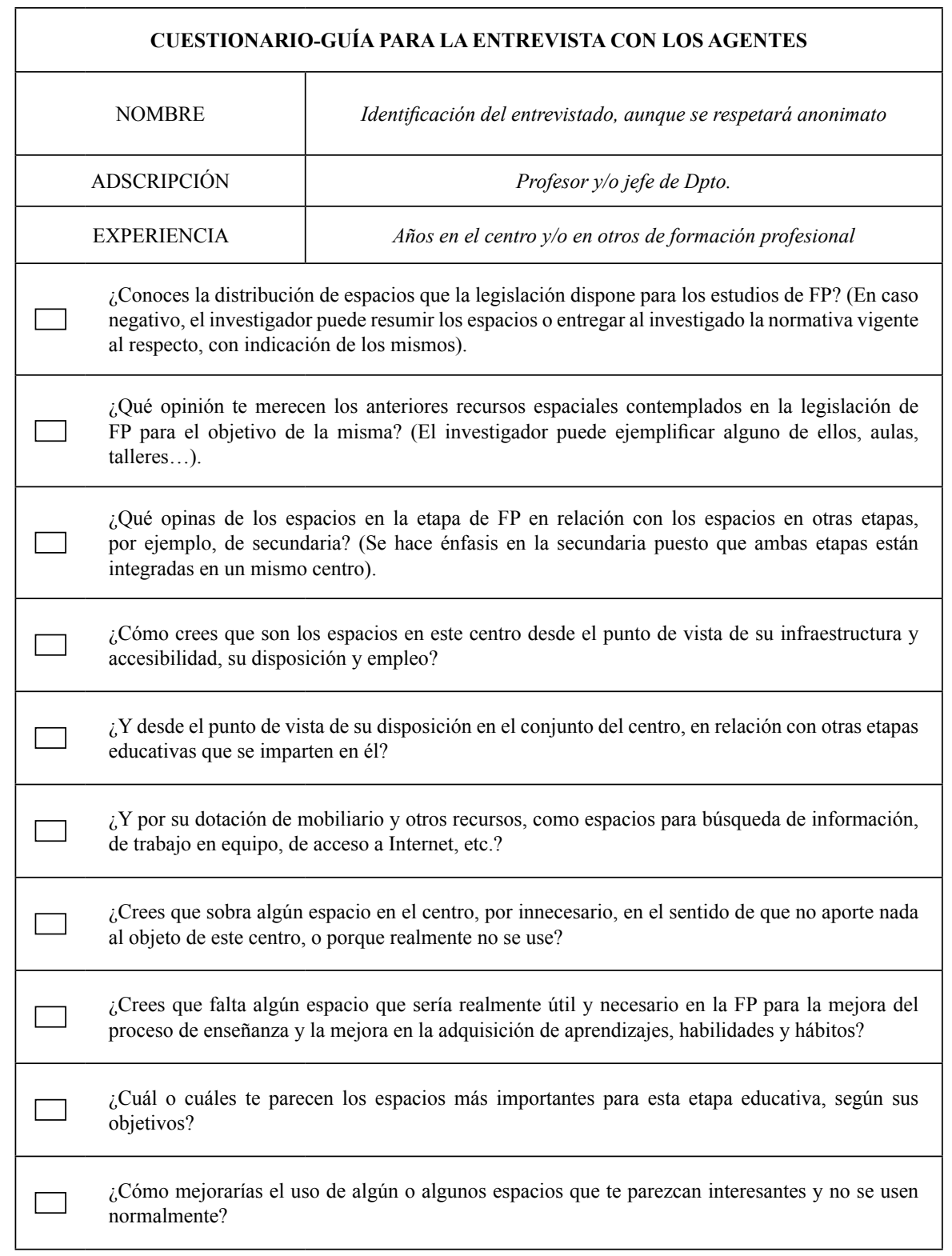

